not significantly different to online FeNO $(\mathrm{p}=0.51)$. Median (IQR) offline FeNO of 5-breaths was 16 (7-22) ppb and 10breaths 13 (5-19) ppb, lower than online FeNO ( $\mathrm{p}=0.03$ and $\mathrm{p}=0.005$ respectively).

The predicted offline values are compared with measured offline values, expressed as a percentage of online, in Table 1.

\begin{tabular}{lllll}
\multicolumn{2}{l}{ Abstract P287 Table 1 } & & \\
\hline & $\begin{array}{l}\text { Predicted FeNO as a } \\
\text { percentage of online }\end{array}$ & $\begin{array}{l}\text { Offline as a percentage of } \\
\text { online FeNO (median) }\end{array}$ & $\begin{array}{l}\text { Interquartile } \\
\text { range }\end{array}$ & $\begin{array}{l}\text { p- } \\
\text { value }\end{array}$ \\
\hline 1 & $94.7 \%$ & $97.2 \%$ & $73.1-120.1 \%$ & 0.81 \\
breath & & $63.6 \%$ & & \\
5 & $73.5 \%$ & $42.9-92.8 \%$ & 0.58 \\
$\begin{array}{l}\text { breaths } \\
10\end{array}$ & $47 \%$ & $43.4 \%$ & & \\
breaths & & & $30.9-67.8 \%$ & 0.41 \\
\hline
\end{tabular}

Conclusion One-breath offline collection methods had comparable results to online FeNO measurements, but higher breath number resulted in lower values likely due to sample contamination with ambient gas and dilution of nitric oxide. These results suggest that multiple breaths should not be used to obtain an offline FeNO result.

\section{P288 PREVALENCE OF NON-PULMONARY EMBOLISM DIAGNOSES ON CT PULMONARY ANGIOGRAPHY. ONE YEAR EXPERIENCE IN A DISTRICT GENERAL HOSPITAL}

${ }^{1} \mathrm{~S}$ Barnes, ${ }^{2} \mathrm{C}$ Ingham, ${ }^{3} \mathrm{~A}$ Pryce, ${ }^{4} \mathrm{R}$ Russell. ${ }^{1}$ Royal Berkshire Hospital, Reading, UK; ${ }^{2}$ Stoke Mandeville Hospital, Aylesbury, UK; ${ }^{3}$ Hammersmith Hospital, London, UK; ${ }^{4}$ Lymington Forest Hospital, Hampshire, UK

\subsection{6/thoraxjnl-2015-207770.424}

Introduction and objectives CT pulmonary angiography (CTPA) is recommended as the investigation of choice for suspected pulmonary embolism (PE). Even in appropriately risk-assessed groups, CTPA often proves negative for PE, but additional diagnostic information is still provided by technically adequate scans. We reviewed our CTPAs to assess technical quality and final diagnosis.

Methods Retrospective review of CTPAs performed in 2013 in a 588 bed district general hospital. All patients selected were considered either intermediate or high probability for PE based on Wells PE clinical risk scoring. Only imaging performed on patients presenting as acute admissions was reviewed. Patients under the age of 16 were excluded, but no upper age limit was applied in order to be fully representative of our patient population and clinical practice. Scans were assessed for their diagnostic and technical quality by the reporting radiologist.

Results 720 CTPAs were selected for review. Patient mean age 66.4 (range 17-103). PE was demonstrated in 135 studies (18.8\%). 111 CTPAs (15\%) excluded PE and were otherwise normal. 355 CTPA (49\%) excluded PE but revealed an alternative diagnosis. Of these, cardiac failure (26\%), emphysema (19\%), pneumonia (16\%), interstitial lung disease (10\%), bronchiectasis (9\%) and pleural disease (8\%) were the most frequently reported clinically significant diagnoses. 119 CTPAs were considered technically inadequate to exclude PE based on insufficient contrast opacification, however an alternative explanatory diagnosis was seen in $76(64 \%)$ of these. In the remaining
43 cases no diagnosis was reported, and only 2 patients had repeat CTPA performed during the same admission. Four patients from the non-diagnostic group represented within a 3 month follow up period and were subsequently proven to have PE on repeat CTPA.

Conclusions CTPA can provide an alternative diagnosis in the majority of cases even if $\mathrm{PE}$ is excluded. Of these, cardiac failure and emphysema were the most common diagnoses. Physicians must be vigilant for non-diagnostic scans and arrange further tests as appropriate, as in our series $4 / 43$ patients with technically inadequate imaging on initial presentation subsequently represented with PE.

\section{P289 ISOLATED MEDIASTINAL AND/OR HILAR LYMPHADENOPATHY: WHAT CAN EBUS-TBNA ADD?}

V Connor, V Tippet, T Kennedy, B Challoner. Aintree University Hospital, Liverpool, UK

\subsection{6/thoraxjnl-2015-207770.425}

Introduction and objectives In recent years, endobronchial ultrasound-guided transbronchial needle aspiration (EBUS-TBNA) has been increasingly used to investigate patients with isolated mediastinal and/or hilar lymphadenopathy (IMHL). There is currently a lack of high quality research to confirm the diagnostic yield and accuracy for these patients.

The aim of this review was to examine the diagnostic yield of EBUS-TBNA in patients with IMHL, to ensure accurate information is being given to patients considering undergoing the procedure.

Methods A retrospective service review was carried out, including consecutive patients listed for EBUS-TBNA between June 2013-June 2014 in a single UK centre.

Results 135 patients were evaluated who underwent EBUSTBNA as a first diagnostic test (full results in Table 1). 35 patients with IMHL underwent EBUS-TBNA within the study period; IMHL was defined as the presence of enlarged mediastinal/hilar lymph nodes on computer tomography (CT) without suggestion of intrathoracic/extra-thoracic malignancy. 34 of these had a diagnostic test (97\%); pathological cause was found in 9 patients (sarcoid $n=7$, malignancy $n=2$ ) with the remaining being diagnosed with reactive lymphadenopathy ( $\mathrm{n}=2574 \%$ ).

This group of 35 patients was sub-classified into those with IMHL with a radiologically suggested diagnosis e.g. sarcoid ( $\mathrm{n}=$ 22) and those with no suggested cause of IMHL ( $\mathrm{n}=13)$. EBUS-TBNA was diagnostic in $95 \%$ and $100 \%$ of patients respectively with the most common diagnosis in both groups remaining that of reactive lymphadenopathy.

Conclusions A significant proportion of patients with IMHL were found to have a diagnosis of reactive lymphadenopathy. With a pathological cause only being found in a minority of cases the appropriateness of EBUS-TBNA to investigate these patients could be questioned.

Our results found a significantly higher prevalence of reactive lymphadenopathy than previously published data; Evinson et al. reported this diagnosis $48 \%$ of patients and the REMEDY trial reported reactive lymphadenopathy in $5 \%$ of patients.

The detection of IMHL and its burden on the health service is likely to increase with increasing use of CT. Due to this a large multi-centred prospective study of patients with IMHL would be advisable to further investigate what the most appropriate strategy to manage these patients should be. 